\title{
Xuesaitong injection as one adjuvant treatment of acute cerebral infarction: a systematic review and meta-analysis
}

Xiaomeng Zhang, Jiarui $\mathrm{Wu}^{*}$ and Bing Zhang ${ }^{*}$

\begin{abstract}
Background: Xuesaitong Injection (XST) is one of the most commonly used medicines for treating acute cerebral infarction (ACI) in China. However, compared to the conventional therapy with western medicines (WM), the effectiveness and safety of XST as an adjuvant treatment for ACI needs to be systematically reviewed.

Methods: Randomized controlled trials (RCTs) comparing XST \& WM with WM for treating ACI were included. Two reviewers independently extracted data. The Cochrane table of Risk of Bias was used to assess the quality of the included studies, and a meta-analysis was conducted using Review Manager 5.2.

Results: 23 RCTs, involving 2196 participants, were included in this study. Methodological quality was not well. The meta-analysis indicated that compared to WM, the combined use of XST and WM was more effective in terms of the total clinical effective rate $[R R=1.21,95 \% \mathrm{Cl}(1.16,1.25), P<0.00001]$, neurological deficit scores $[M D=-3.31$, $95 \% \mathrm{Cl}(-4.10,-2.52), P<0.00001]$, and plasma viscosity $[M D=-0.13,95 \% \mathrm{Cl}(-0.15,-0.11), P<0.00001]$. The included studies reported 37 adverse events, 17 of which belonged to experimental groups.

Conclusion: XST combined with WM appeared to be effective for ACl. However, the evidence of XST for treating $\mathrm{ACl}$ should be carefully interpreted due to the small sample size, limited number of trials, and unsatisfactory quality of research.
\end{abstract}

Keywords: Xuesaitong injection (XST), Acute cerebral infarction (ACI), Systematic review, Meta-analysis

\section{Background}

Acute cerebral infarction (ACI) is one of the most commonly seen cerebral vascular diseases, which accounting for about $70 \%$ of stroke [1]. The incidence, mortality and recurrence rate of $\mathrm{ACI}$ are both high, usually leads to serious damage of central nervous system [2]. ACI refers to the process that artery stenosis or blockage causing brain tissue hypoxic ischemia, and then resulting in brain dysfunction [3]. Currently, the conventional therapy with western medicines (WM) mainly includes thrombolysis, improving microcirculation, restoring blood supply to ischemic area, using cerebral protection agents, controlling cerebral edema, preventing and treating complications, controlling hypertension, reducing blood viscosity, and so on [1,2].

\footnotetext{
*Correspondence: exogamy@163.com; zhangbing6@263.com Department of Clinical Chinese Pharmacy, School of Chinese Materia Medica, Beijing University of Chinese Medicine, Beijing 100102, China
}

Some scholars hold the opinion that in traditional Chinese medicine (TCM), the pathogenesis of $\mathrm{ACI}$ is due to the disorder of Qi and blood, and then cerebral veins blocking, brain cells ischemia and hypoxia [4]. Therefore, the treatment of promoting blood flow is the first choice. There are numbers of literatures reported that Chinese medicinal herbs have unique advantages in the treatment of ACI by removing stasis. Xuesaitong Injection (XST), as one of the most commonly used medicines for treating ACI in China. Its active ingredient is attributed to extract from the roots of Pannax notoginseng (Sanqi), which mainly contains tetracyclic triterpenoid saponins, generally called as "Panax notoginseng saponins" (PNS). PNS has good therapeutic effects on cardiovascular and cerebrovascular system, blood system, and nervous system. Some pharmacological studies also showed that XST can reduce the edema caused by cerebral ischemia, protect vascular endothelial cells, inhibit the adhesion and aggregation of 
platelet, dissolve thrombus, eliminate superoxide anion radicals, and so on [5-9], which are all beneficial for treating acute stroke [10].

There were two systematic reviews regarding XST in the treatment of cerebral infarction [11,12], both showing the superiority of XST to control group, their delimiting of acute stage, unreasonable merging of intervention measures, and low quality of literatures still being controversial. Accordingly, in this systematic review, we chose the published, qualified and well homogeneity clinical studies regarding the combined use of XST for treating ACI to make meta-analysis, hopefully to provide more reliable evidence for XST's clinical application.

\section{Methods}

\section{Inclusion criteria}

(1) Study type: clinical randomized controlled trials (RCTs) using XST as the adjuvant treatment of ACI, regardless of blinding. (2) Participants: The diagnostic criterion in terms of TCM was "Apoplexy diagnostic efficacy assessment standards"; that used in terms of WM was "various types of cerebrovascular disease diagnostic points", as determined after 1995 [13]. Diagnoses were validated using computed tomography or magnetic resonance imaging scanning. The course of disease was in 3 days or shorter, and all participants were experiencing the first onset of ACI, no limits on age, gender, race or severity of disease. (3) Interventions: RCTs comparing combined therapy of XST and conventional treatments versus conventional treatments alone were included. Conventional treatments included thrombolytic therapy, cerebral protection agents, and so on. The drugs could be Dextran-40, Mannitol, Aspirin, Citicoline, Venoruton, Defibrase, Sodium ozagrel, et al. As long as the conventional treatments were the same between study groups in one RCT, the RCT could be included. No limitation on the dosages and treatment courses. (4) Outcomes: Our primary outcome measure was the total clinical effective rate, using the following formula: total clinical effective rate $(\%)=$ (number of recovered patients + number of patients with significant progress + number of patients with progress) / total number $x$ $100 \%$. Efficacy criteria predominantly referred to the reduction of neurological deficit score. Recovered was determined when the neurological deficit score decreased from $91 \%$ to $100 \%$. Significant progress was determined when the neurological deficit score decreased by between $46 \%$ and $90 \%$. Progress was determined when the neurological deficit score decreased by between $18 \%$ and $45 \%$. No change or worsen was determined when the functional deficit score decreased by $<17 \%$. As secondary outcomes, we compared neurological deficit score, hemorheology value, and number of adverse drug reactions (ADR)/adverse events (AE).

\section{Exclusion criteria}

If involved any condition of the followings, trials can be ruled out: data was incorrect, incomplete or not available; patients with severe cognitive disorder, or hemorrhagic tendency, or serious complications, such as atrial fibrillation, severe heart failure, severe liver and kidney diseases; undergoing surgery, acupuncture or other physical therapy; combined with any herbal medicines in experimental or control group during the treatment.

\section{Searching strategies}

The following databases were used for search: the China National Knowledge Infrastructure Database (CNKI, 980-2014.5), Wan fang Database (1990-2014.5), China Science and Technology Journal Database (VIP, 19892014.5), Chinese Biomedical Literature Database (CBM, 1981-2014.5), PubMed (1990-2014.5), Embase (19902014.5), and the Cochrane Library (1990-2014.5).

We combined different search strategies as follows: for English databases, we used free text terms as "Xuesaitong" and "acute cerebral infarction"; for Chinese databases, we used subject terms as "Xuesaitong Zhu She Ye" or "Zhu She Yong Xuesaitong "or "Xuesaitong Dong Gan Fen Zhen" or "Luotai", and then "Que Xue Xing Cu Zhong" or "Que Xue Xing Zhong Feng" or "Que Xue Xing Nao Xue Guan Bing" or "Nao Geng Si" or "Nao Geng Se" for secondary retrieval. No language restriction was used.

\section{Data extraction and quality assessment}

Two reviewers (XZ and JW) independently screened trials. If there was incomplete information in the study, the reviewer would firstly contact original authors via e-mail or telephone. If no response, the reviewer would make decision on including or excluding based on the importance of lack information. XZ and JW independently extracted data, including basic information of patients, interventions, duration of treatment, outcomes and methodological quality. And the risk of bias of the included trials was assessed according to the Cochrane risk of bias tool [14]. It assessed the risk of bias of random sequence generation, allocation concealment, blinding of participants and personnel, blinding of outcome assessment, incomplete outcome data, selective reporting, and other biases. Judgments were given for each item as: low, unclear, and high risk of bias. XZ and JW independently completed and mutually checked the allocated grades. Any disagreements on data extraction and quality assessment were resolved by consensus, or if required by a third reviewer.

\section{Data synthesis}

RevMan 5.2 software was used, whose package was produced and updated by the Nordic Cochrane Centre. 
Relative risk $(R R)$ was used for dichotomous data, and mean difference $(M D)$ was used for continuous variables, both with 95\% confidence interval (95\% CI), $P<0.05$ was considered statistically significant between experiment and control group. The Chi-square test was used for checking the heterogeneity between studies, and $I^{2}$ was used to show the size of heterogeneity. If $P>0.1$ and $I^{2}<50 \%$, there was determined to be little heterogeneity between studies, then we used a fixed effect model, otherwise we should use a random effect model. If the number of included trials was sufficient, a funnel plot would be carried out to assess publication bias. Sensitivity analysis was performed to inspect the robustness of the result.

\section{Results}

Searching result

Total 1181 articles were retrieved from the databases listed above. Finally, a total of 23 RCTs were included [15-37] (Figure 1 flow chart of literature search).

\section{Studies description}

The included studies were published between 1999 and 2013. All RCTs originated from China, and were published in Chinese. Altogether, 23 RCTs included a total of 2196 patients. As presented in Table 1, the experimental group consisted of 1141 patients, while the control group 1055. The included patients were primarily elderly, the average age of which was about 60.5 , with a

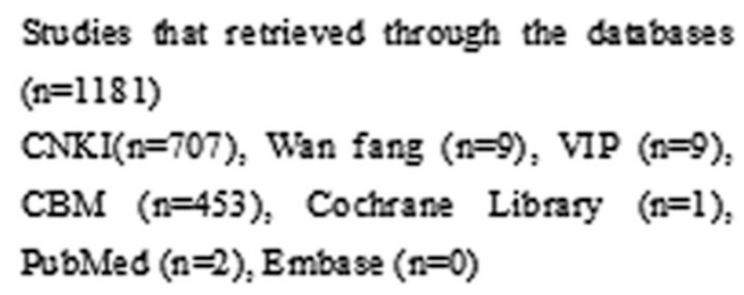

Studies obtained through other resources $(n=0)$

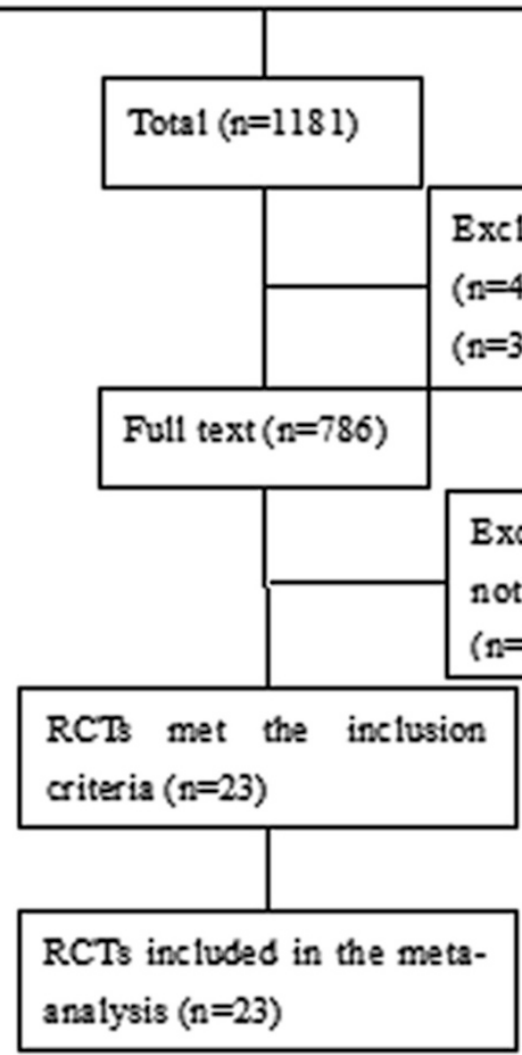

Figure 1 Flow chart of literature search. 
Table 1 Characteristics of included trials on XST for ACI

\begin{tabular}{|c|c|c|c|c|c|c|c|c|c|}
\hline \multirow[t]{2}{*}{ Study ID } & \multirow{2}{*}{$\begin{array}{l}\text { Sex } \\
(M / F)\end{array}$} & \multirow[t]{2}{*}{ Age (a) } & \multirow{2}{*}{$\begin{array}{l}\text { Course } \\
\text { of disease (h) }\end{array}$} & \multirow[t]{2}{*}{$N(E / C)$} & \multicolumn{2}{|l|}{ Intervention } & \multirow{2}{*}{$\begin{array}{l}\text { Duration } \\
\text { (days) }\end{array}$} & \multirow[t]{2}{*}{ Outcomes } & \multirow[t]{2}{*}{ ADR/ADE } \\
\hline & & & & & Experimental & Control & & & \\
\hline Li 1999 [15] & $29 / 33$ & $62.9 \pm 7.1(57 \sim 71)$ & $\leq 48$ & $31 / 31$ & XST 400 mg + WM & WM (Defibrase et al.) & 14 & $\begin{array}{l}\text { Total effective rate, changes in cerebral } \\
\text { blood flow and hemorheology }\end{array}$ & Unclear \\
\hline Zhang 2002 [16] & $52 / 48$ & $53.0(37 \sim 70)$ & $\leq 72$ & $60 / 40$ & Luotai $400 \mathrm{mg}+\mathrm{WM}$ & Xiaoshuanling + WM & 14 & Total effective rate & Unclear \\
\hline Yuan 2003 [17] & $37 / 25$ & $42 \sim 79$ & $\leq 72$ & $32 / 30$ & XST $20 \mathrm{ml}+$ WM & WM (Piracetam et al.) & 15 & $\begin{array}{l}\text { Total effective rate, changes } \\
\text { in hemorheology }\end{array}$ & Unclear \\
\hline Li 2003 [18] & $69 / 27$ & $55.8(42 \sim 71)$ & $\leq 72$ & $48 / 48$ & XST $400 \mathrm{mg}$ & WM (Dextran $40+$ citicoline) & 15 & Total effective rate & Unclear \\
\hline Li 2005 [19] & - & $48 \sim 73$ & $\leq 72$ & $80 / 76$ & XST $200 \mathrm{mg}+$ WM & WM & 20 & Total effective rate & E 3 \\
\hline He 2006 [20] & $48 / 32$ & $59.3 \pm 12.1(46 \sim 80)$ & $\leq 72$ & $40 / 40$ & XST $10 \mathrm{ml}+$ WM & Dextran $40+$ WM & 14 & $\begin{array}{l}\text { Total effective rate, neurological } \\
\text { deficit score }\end{array}$ & None \\
\hline Wang 2006 [21] & $34 / 21$ & $63.8(39 \sim 79)$ & $\leq 24$ & $36 / 19$ & XST 600 mg + WM & Venoruton + WM & 21 & Total effective rate & None \\
\hline \multirow[t]{2}{*}{ Yuan 2006 [22] } & \multirow[t]{2}{*}{$64 / 31$} & \multirow[t]{2}{*}{$63.0 \pm 10.4(46 \sim 74)$} & \multirow[t]{2}{*}{$\leq 24$} & \multirow[t]{2}{*}{$49 / 46$} & \multirow[t]{2}{*}{ XST $250 \sim 500 \mathrm{mg}+\mathrm{WM}$} & \multirow[t]{2}{*}{ WM (Defibrase et al.) } & \multirow[t]{2}{*}{15} & \multirow[t]{2}{*}{ Total effective rate } & E 3 \\
\hline & & & & & & & & & C 7 \\
\hline Zhao 2006 [23] & $43 / 38$ & $60.9 \pm 8.1(45 \sim 70)$ & $\leq 72$ & $40 / 41$ & XST $10 \mathrm{ml}+$ WM & $\begin{array}{l}\text { WM (Sodium ozagrel + } \\
\text { Defibrase et al.) }\end{array}$ & 14 & $\begin{array}{l}\text { Total effective rate, changes } \\
\text { in hemorheology }\end{array}$ & Unclear \\
\hline Li 2007 [24] & $54 / 36$ & $59.5 \pm 13.2(48 \sim 79)$ & $\leq 48$ & $45 / 45$ & XST $10 \mathrm{ml}+$ WM & Dextran $40+W M$ & 15 & Total effective rate & None \\
\hline Wang 2007A [25] & $50 / 26$ & $63.6(39 \sim 82)$ & $\leq 24$ & $50 / 26$ & XST $600 \mathrm{mg}+\mathrm{WM}$ & Venoruton + WM & 21 & Total effective rate & None \\
\hline Wang 2007B [26] & - & $69.4(43 \sim 80)$ & $\leq 72$ & $30 / 30$ & XST $20 \mathrm{ml}+$ WM & WM (Venoruton et al.) & 14 & Total effective rate & None \\
\hline Rong 2008 [27] & $50 / 46$ & $59.1 \pm 8.4(39 \sim 77)$ & $\leq 72$ & $51 / 45$ & XST 400 mg + WM & Venoruton + WM & 14 & $\begin{array}{l}\text { Total effective rate, neurological } \\
\text { deficit score, Modified Barthel Index, } \\
\text { changes in hemorheology }\end{array}$ & None \\
\hline Zhang 2008 [28] & $70 / 50$ & $56 \pm 11(48 \sim 78)$ & $\leq 48$ & $65 / 65$ & XST $200 \sim 400 \mathrm{mg}+\mathrm{WM}$ & WM & 15 & $\begin{array}{l}\text { Total effective rate, changes in } \\
\text { hemorheology }\end{array}$ & Unclear \\
\hline Zi 2008 [29] & $49 / 33$ & $59.5 \pm 13.2(48 \sim 79)$ & $\leq 48$ & $41 / 41$ & XST $10 \mathrm{ml}+$ WM & Dextran $40+W M$ & 15 & $\begin{array}{l}\text { Total effective rate, neurological } \\
\text { deficit score }\end{array}$ & None \\
\hline Duan 2009 [30] & $35 / 34$ & $64.5 \pm 8.2(33 \sim 75)$ & $\leq 48$ & $36 / 33$ & XST 500 mg + WM & WM & 14 & Total effective rate & None \\
\hline \multirow[t]{2}{*}{ Ma 2009 [31] } & \multirow[t]{2}{*}{$117 / 83$} & \multirow[t]{2}{*}{$65.1 \pm 7.0(45 \sim 85)$} & \multirow[t]{2}{*}{$\leq 72$} & \multirow[t]{2}{*}{$100 / 100$} & \multirow[t]{2}{*}{ XST $400 \mathrm{mg}+\mathrm{WM}$} & \multirow[t]{2}{*}{ Venoruton + WM } & \multirow[t]{2}{*}{15} & \multirow{2}{*}{$\begin{array}{l}\text { Total effective rate, neurological } \\
\text { deficit score, changes in } \\
\text { hemorheology, glycemia and lipidemia }\end{array}$} & E 9 \\
\hline & & & & & & & & & C 10 \\
\hline \multirow[t]{2}{*}{ Cai 2011 [32] } & \multirow[t]{2}{*}{$42 / 18$} & \multirow[t]{2}{*}{$64.2(47 \sim 86)$} & \multirow[t]{2}{*}{$\leq 48$} & \multirow[t]{2}{*}{$30 / 30$} & XST 400 mg + WM & WM (Buflomedil hydrochloride + & 14 & Total effective rate, neurological & E 2 \\
\hline & & & & & & & & & C 1 \\
\hline Fu 2011 [33] & $62 / 58$ & $55.4 \pm 5.1$ & $\leq 48$ & $64 / 58$ & XST 400 mg + WM & WM (Sodium ozagrel et al.) & 14 & $\begin{array}{l}\text { Total effective rate, neurological } \\
\text { deficit score }\end{array}$ & None \\
\hline He 2011 [34] & $57 / 65$ & $59 \sim 78$ & $\leq 72$ & $62 / 60$ & XST 200 mg + WM & WM (Citicoline, Aspirin) & 14 & $\begin{array}{l}\text { Total effective rate, neurological } \\
\text { deficit score }\end{array}$ & None \\
\hline
\end{tabular}


Table 1 Characteristics of included trials on XST for ACI (Continued)

\begin{tabular}{|c|c|c|c|c|c|c|c|c|c|}
\hline Wang 2011 [35] & $45 / 37$ & $55.87 \pm 5.23(42 \sim 76)$ & $\leq 19$ & $41 / 41$ & XST 400 mg + WM & WM & 21 & Total effective rate, $\mathrm{Hs}$-CRP & Unclear \\
\hline Yang 2012 [36] & $33 / 27$ & $57 \pm 4(47 \sim 68)$ & $\leq 48$ & $30 / 30$ & XST 400 mg + WM & WM (Sodium ozagrel et al.) & 14 & Total effective rate & C 2 \\
\hline Song 2013 [37] & $117 / 43$ & 62.1 & $\leq 24$ & $80 / 80$ & XST 400 mg + WM & WM & 15 & Total effective rate & Unclear \\
\hline
\end{tabular}

NOTE: M: Males; F: Females; E: Experimental group; C: Control group; ADR: Adverse drug reactions; ADE: Adverse drug events; XST: Xuesaitong Injection; WM: Conventional therapy with western medicines, such as

Dextran-40, Mannitol, Aspirin, Citicoline, Venoruton, Defibrase, Sodium ozagrel, et al. 
range of 33 to 86 years old. $58.4 \%$ of the participants were males. In the experimental group, all the RCTs used XST combined with the same WM as control group. The daily dose of XST ranged from $200 \sim 600 \mathrm{mg}$. In the included RCTs, XST was produced by Xing Zhong Pharmaceutical Co., Ltd. in Kunming, Plant Pharmaceutical Co., Ltd. in Yunnan Province, China, or Zhenbaodao Pharmaceutical Co., Ltd. in Heilongiiang Province, China. The duration of treatments ranged from 14 days to 28 days. More details were shown in Table 1.

\section{Quality of the included studies}

Three trials used a random number table to generate random sequence $[26,32,34]$, two grouped according to the time of admission $[21,35]$, and one grouped according to odd and even numbers [24]. And there were two trials may be used non-random sequence $[16,36]$. Only one trail performed single-blinding [18]. Others in the included trials in this meta-analysis were judged as unclear random sequence generation, inadequate allocation concealment, and inadequate blinding. None of the included trials had incomplete outcome data or selective reporting. Therefore, the quality of the included studies was not well. More details of the trials were presented in Figure 2. Funnel plot analysis showed that there was some significant publication bias in the comparison, as shown in Figure 3.

\section{Effects of interventions}

\section{Total clinical effective rate}

All the included studies [15-37] reported the total effective rate. The meta-analysis showed that the effect of XST combined with WM was better than WM alone in improving the total effective rate. The statistical difference between the two groups was significant [23RCTs, $I^{2}=25 \%$, fixed effect model, $R R=1.21,95 \% C I(1.16,1.25), P<0.00001$ ]. (As shown in Figure 4).

\section{Sensitivity analysis}

To confirm the stability of the result of the total clinical effective rate, we respectively removed the most and the least weighted, and changed from fixed mode to random mode. After removing the most weighted (Ma et al. [31]), the result was $R R=1.21$ [95\% CI $(1.17,1.26), P<0.00001]$. The result of removing the least ( $\mathrm{Li}$ et al. [15] and Wang et al. [21]) was $R R=1.20$ [95\% $C I(1.16,1.25), P<0.00001]$. The result of changing the mode was $R R=1.18$ [95\% CI (1.13, 1.23), $P<0.00001]$. There was no obviously diversity, so the degree of the sensitivity of the study was not high.

\section{Neurological deficit score}

There were seven trials mentioned neurological deficit score $[20,27,28,31-34]$. Heterogeneity between studies was large $\left(P<0.00001, I^{2}=84>25 \%\right)$, we should use a random effect model. The result showed that XST was more effective in reducing neurological deficit score. The statistical difference between the two groups was significant $[M D=-4.35,95 \% C I(-6.61,-2.08), P=0.0002]$. (Shown in Figure 5).

\section{Plasma viscosity}

There were five trials mentioned the content of plasma viscosity $[15,17,23,28,31]$. The result showed that XST was more effective in reducing the content of plasma viscosity. The statistical difference between two groups was significant [5 RCTs, $I^{2}=77 \%$, random effect model, $M D=-0.14,95 \% C I(-0.21,-0.08), P<0.00001]$. (Shown in Figure 6).

\section{Adverse events}

Five trials [19,21,32,33,37] reported 35 cases of AE. None of participants who reported AE developed bleeding or liver and kidney damage, and all participants with $\mathrm{AE}$ recovered after symptomatic treatments. The occurrence of AE did not affect treatment process. There were 17 from 35 cases occurred in experimental group, which were manifested as two cases of headache and nausea, 


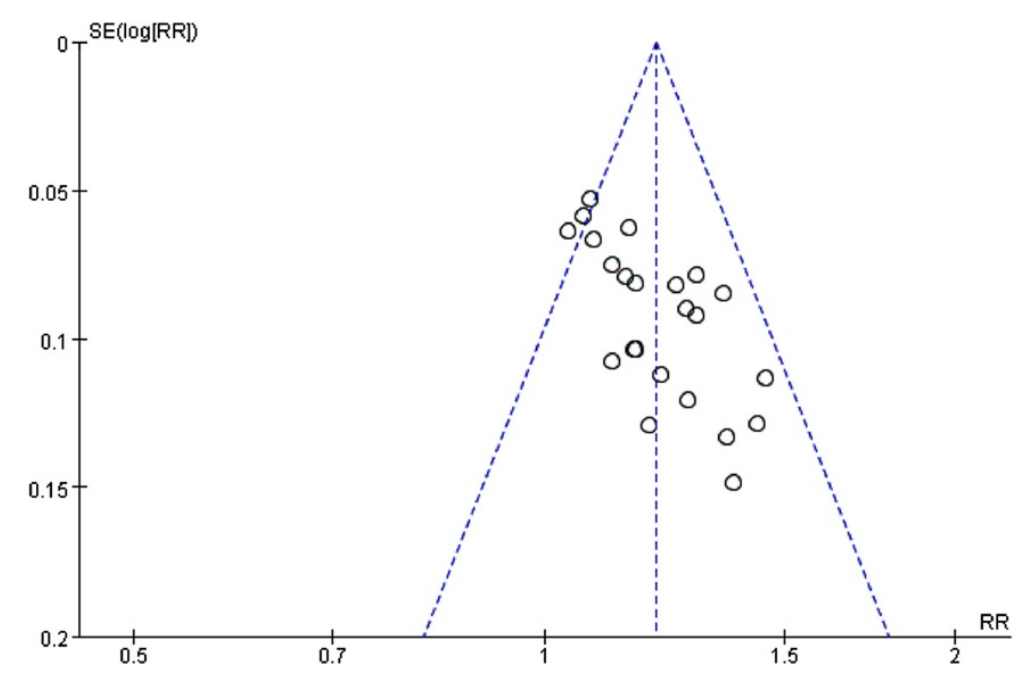

Figure 3 Funnel plot of the total effective rate of XST in the treatment of ACI. XST: Xuesaitong Injection; ACl: acute cerebral infarction.

three cases of rash, three cases of a small amount of bleeding points in subcutaneous, four cases of dizziness, palpitation, and five cases of flush. While 20 cases occurred in control group, which were manifested as one case of bleeding gums, one case of gastrointestinal bleeding, three cases of nausea and vomiting, four cases of dizziness and palpitation, five cases of bleeding points in subcutaneous, and six cases of face red. Ten trials clearly expressed that no ADRs/ADEs occurred in their studies [20,21,25-28,30,31,34,35]. And the other eight trials provided no information.

\section{Discussion}

\section{Discussion on the systematic review}

Following the systematic review, we can summarize that $\mathrm{XST}$ as an adjuvant treatment for WM for ACI was more effective. It could increase the total clinical effective rate, decrease the degree of neurological deficit, and reduce the content of plasma viscosity, which were consistent with results of previous system reviews $[11,12]$. Compared with previously reported data, we were not only adding more high-quality, small heterogeneous literatures, but also strictly controlling the course of disease and the medications of control group. Therefore, the present systematic review was more scientific and precise. But regarding outcome of neurological deficit scores, Cai 2011 [32] reported large heterogeneity with the other included studies, which may be related to less effectiveness of Buflomedil Hydrochloride and low molecular heparin on improving neurological deficit. And though the efficacy of XST for treating ACI is sure, its safety remains to be further assessed. Accordingly, from

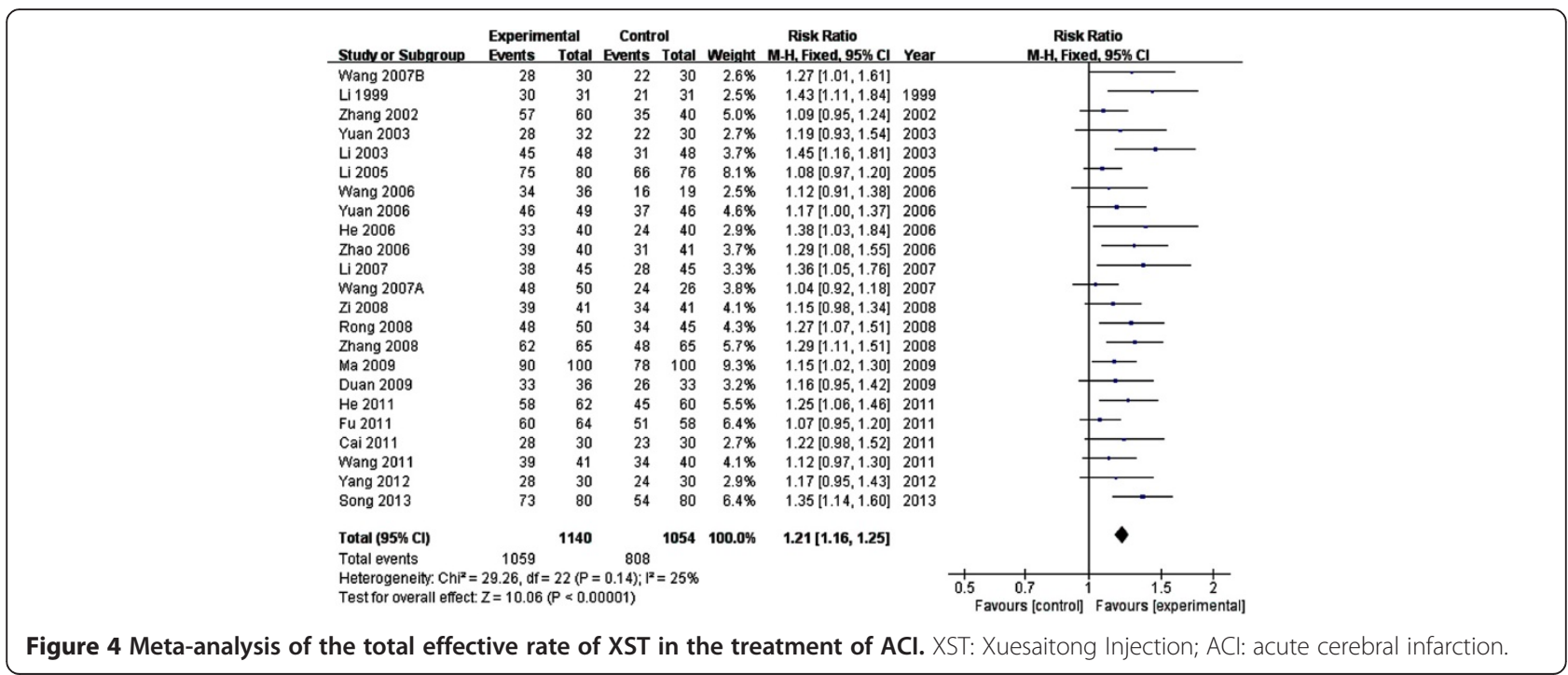




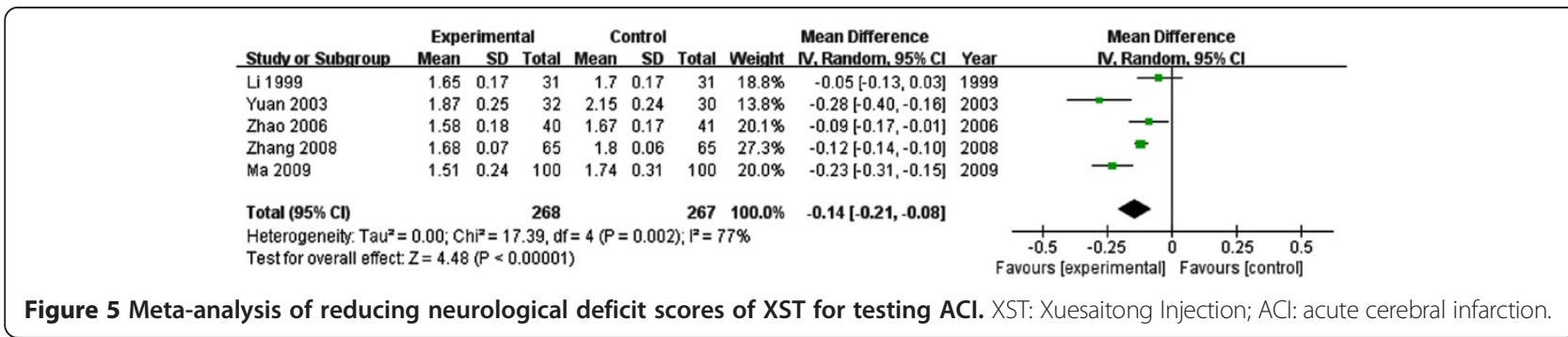

the existing clinical evidence, XST can be promoted using in clinical to form an integrative therapy.

Recommendation on the efficacy evaluation of XST in the treatment of $\mathrm{ACl}$

A research from Asia Pacific Region found that significant correlation exists between dyslipidemia and occurrence of ischemic stroke. When the total cholesterol (TC) increased by $1 \mathrm{mmol} / \mathrm{L}$, the incidence of stroke will increase by $25 \%$ [38-40]. Meanwhile, experiments demonstrated that PNS, the main ingredient in XST, can significantly reduce blood lipids and lipid peroxidation products [41]. In the systematic review, only one study [31] monitored the changes in TC was $M D=-1.01[95 \%$ $C I(-1.41,-0.61), P<0.00001)]$, which had significant differences between two groups. Accordingly, depending on the GRADE methods, we propose that blood lipids level should be used as one of the important indicators for the evaluation of efficacy of XST for treating ACI in the further clinical studies.

On the other hand, high C-reactive protein (Hs-CRP), as a kind of acute phase proteins, may increase rapidly when suffering various acute inflammations, tissue damage, and other diseases. When patients improved, the content of Hs-CRP quickly returned to normal. Its increasing has positive correlation with the degree of infection, and it was with high sensitivity [42]. Accordingly, Hs-CRP can be as an independent risk factor for cerebral infarction to predict the severity of cerebral infarction and infarct size.
But in the included studies, only 1 trail [35] compared the changes in Hs-CRP, which result was $M D=-6.62$ [95\% CI (-8.29, -4.95), $P<0.00001]$. In sum, depending on the GRADE methods, we considered Hs-CRP can also serve as one of important indicators in evaluation of efficacy of ACI.

\section{XST'ADRs}

In clinic, ADR of XST's was reported in large quantity. The common symptoms included headache, dry throat, palpitation, pruritus, severe rash, and the severe even have anaphylactic shock [43], which are generally in consistent with the AEs that reported in our meta-analysis. Although there were no serious ADRs, we could not conclude on its safety yet. Therefore, special attention should be paid to the observation of AE. The metaanalysis showed that there were four studies [21,22,25,30], in which their dose of XST exceeded $200 \sim 400 \mathrm{mg}$, and such high dose may easily cause ADRs according to instruction manual. In addition, there were also one study [44] showed that allergies occurrence time was mostly to exceed $7 \mathrm{~d}$. It prompts that medical staffs need to have a properly assessment on efficacy to avoid overdose. Patients with allergic history have more chance to suffer ADR, so the clinicians need to know well the allergic history of patients. Furthermore, drug combination is also a high risk for ADR occurrence; accordingly, it should reduce the quantity of drugs as few as possible for combination use.

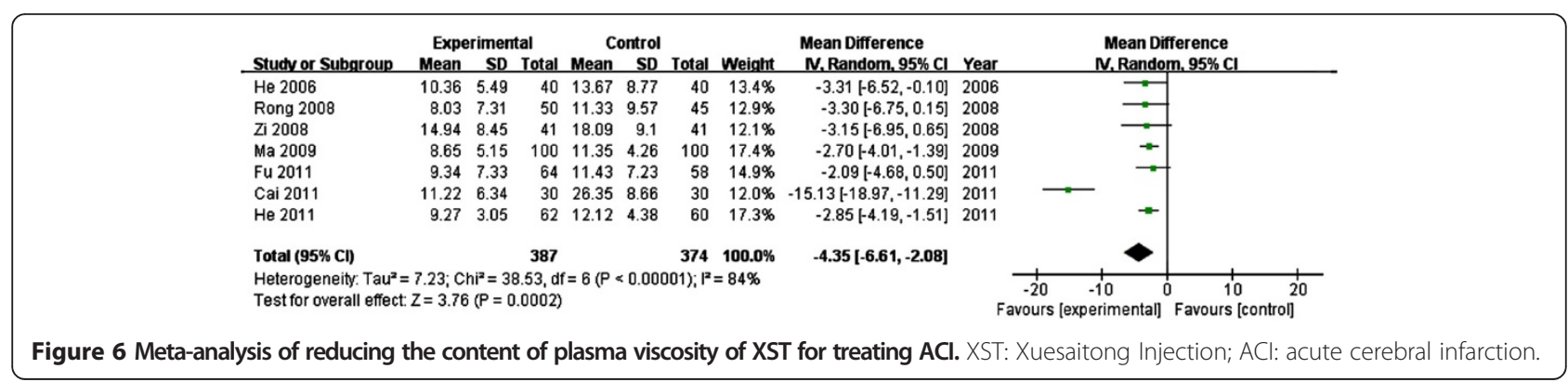




\section{Limitation of this systematic review and direction for further clinical research}

Although the systematic review showed that XST as adjuvant treatment for WM for ACI was effective, the methodological quality of included studies was not ideal. Only 6 studies described random allocation method, and none of them described allocation concealment. Only one study used single-blinding. All the RCTs had small sample size. In the included studies, XST was products from two manufacturers and the dosage of XST varied a lot. In addition, the systematic review itself also has some limitations. Since all trials were published in Chinese, we could not exclude the potential publication bias. More rigorously designed RCTs are needed to confirm the effectiveness of XST for ACI.

\section{Conclusion}

According to low quality evidences, XST as an adjuvant treatment for conventional treatments for treating ACI was beneficial comparing to conventional treatments alone. Although the safety of XST requires further research, it is worthy to be promoted using in clinical.

\section{Abbreviations}

ACl: Acute cerebral infarction; XST: Xuesaitong injection; WM: Conventional treatment with western medicine; TCM: Traditional Chinese medicine; RCTs: Randomized controlled trials; CNKI: China national knowledge infrastructure; VIP: China science and technology journal database; CBM: Chinese biomedical literature database; ADRs: Adverse drug reactions; ADEs: Adverse drug events; RR: Relative risk; MD: Mean difference; 95\% Cl: 95\% confidence interval.

\section{Competing interests}

The authors declare that they have no competing interests.

\section{Authors' contributions}

XZ conducted the database search, assessed studies for inclusion, extracted and analyzed the data, and prepared the manuscript draft. JW conceived the study, and conducted the database search, assessed studies for inclusion, extracted the data which followed by cross checking with $X Z$, and revised the manuscript. BZ conceived the study, analyzed the data, and revised the manuscript. All authors read and approved the final version of the manuscript.

\section{Acknowledgements}

The study was financially supported by National Natural Science Foundation of China (No. 81473547); National Science and Technology Support Program of China (No. 2006BAI21B11); Scientific Research Innovation Team Project of Beijing University of Chinese Medicine (No.2011-CXTD-14), and Postgraduate Independent Topics (No. 2014-JYBZZ-XS-078).

Received: 3 September 2014 Accepted: 18 February 2015

Published online: 27 February 2015

\section{References}

1. Luo JN. New progress on treating acute cerebral infarction. Pract J Cardiac Cereb Pneumal Vasc Dis. 2010;18(10):1546-7.

2. Deng $L$, Liu XD, Zhang YB, Li JM. Advances in the treatment of acute cerebral infarction. Chin Gen Pract. 2011;14(3B):825-9.

3. Zhou K. Progress in the treatment of acute cerebral infarction. Pract J Card Cereb Pneumal Vasc Dis. 2010;18(11):1726-8

4. Su ZQ, Zhang YX, Yang N, Tao JP, Kang B. Preliminary analysis on TCM pathogenesis of acute cerebral infarction. J Liaoning Univ TCM. 2014;16(3):175-7.
5. Lin CR. The literature and clinical research of xuesaitong injection to treat acute cerebral infarction. Chinese Medicine: Guangzhou University of; 2007

6. Yang ZG, Chen EQ, Yu SD, Sun HX. Recent advances in the research of pharmacological activities of Panax notoginseng saponins. Chin J Vet Drug. 2005;39(1):33-7.

7. Xiao RM, Qiu XQ, Su JF. New progress of pharmacological study of panax notoginseng saponin for treating cardiovascular disease. China Healthcare Innov. 2009;4(11):30-1.

8. Yin CQ, Deng HS, Cheng J. Overview of the pharmacological effects of panax notoginseng saponin for blood, cardiovascular and central nervous system. Sci Technol Inf. 2010;29:50-1.

9. Zhu HC, Guo JJ, Ren Y. Progress of pharmacological researches of panax notoginseng saponin. Henan Tradit Chin Med. 2014;34(3):537-41.

10. Zhang SS. Comparative efficacy advantage of panax preparations in treating different types of stroke based on a systematic review. J Shandong Univ Tradit Chin Med. 2013;37(2):91-3.

11. Ding $X$. Meta analysis of xuesaitong injection for the treatment of acute cerehral infarction. Strait Pharm J. 2009;21(10):93-6.

12. Wei XC, Zhu LQ. Meta analysis of xuesaitong injection for the treatment of cerehral infarction. Tianjin Pharm. 2010;22(4):25-8.

13. Chinese society for Neuroscience, Chinese society for neurosurgery. Diagnosis of various types of cerebrovascular disease. Chin J Neurol. 1996:29(6):379.

14. Higgins JPT, Altman DG, Gøtzsche PC, Jüni P, Moher D, Oxman AD, et al. The cochrane collaboration's tool for assessing risk of bias in randomised trials. BMJ. 2011;343:d5928.

15. Li F, GU DX, Li YJ, Shi JM. Observation on curative effects of treatment with panax notoginsenoside injection combined with batroxobin for acutely cerebral infarction in 31 patients. Chin J Integr Tradit Western Med Intensive Crit Care. 1999;6(10):470-2.

16. Zhang LJ, Zhang SS. Observation on effect of 60 cases of Luotai in the treatment of cerebral infarction. J Huaihai Med. 2003;20(1):79.

17. Yuan L. Observation on 34 cases of xuesaitong injection in the treatment of acute cerebral infarction. J Emerg Tradit Chin Med. 2003;12(2):133-45.

18. Li YS. Effect of 48 cases of xuesaitong injection on treating acute cerebral infarction. China Hydropower Med. 2003;15(5):293.

19. Li K, Qing XH. Observation on effect of xuesaitong injection on treating acute cerebral infarction. J Clin Med Pract. 2005;9(11):93-4.

20. He RZ. Effect of xuesaitong injection on treating 40 patients with acute cerebral infarction. Hebei Med. 2006;12(8):756-8.

21. Wang WC. Xuesaitong injection in the treatment of acute cerebral infarction. J Med Forum. 2006;27(15):56.

22. Yuan QD, Jiang JL. Observation on 49 cases of xuesaitong injection combined with defibrase in treating with acute cerebral infarction. J Snake. 2006;18(1):20-1.

23. Zhao BR. Observation on 40 cases of xuesaitong injection combined with defibrase and qinger injection in the treatment of acute cerebral infarction. Chin Med Guides. 2006;3(11):63-4.

24. Li MF, Jiang YB. Clinical observation on xuesaitong injection in treatment of patients with acute cerebral infarction. Mod Med J China. 2007;9(10):93-4.

25. Wang QR. Xuesaitong injection on treating acute cerebral infarction. J Med Forum. 2007;28(20):59.

26. Wang W, Li HJ. Observation on the effect of xuesaitong injection in the treatment of acute cerebral infarction. J Med Forum. 2007;28(14):63.

27. Rong Z, Zhi HP. Case control study of treatment of acute cerebral infarction with xuesaitong injection. J Neurol Neurorehabil. 2008;5(2):85-6. 96.

28. Zhang A, Zhang XR. Clinical study on xuesaitong injection in the treatment of acute cerebral infarction. J Pract Med Tech. 2008;15(19):2508-9.

29. Zi JF, Yue YL, Ma FS. Clinical observation on xuesaitong injection in treating acute cerebral infarction. Chin J Misdiagnostics. 2008;8(30):7353-4

30. Duan $\mathrm{H}, \mathrm{Ai}$ M. Observation on the effect of 69 cases of xuesaitong injection in treatment of acute cerebral infarction. Pract J Card Cereb Pneumal Vascular Dis. 2009;17(3):192.

31. Ma CC, Wang RG. Observation on the effect of 100 cases of xuesaitong injection in the treatment of acute cerebral infarction. Chin J Coal Ind Med. 2009;12(4):595-6.

32. Cai SL. Observation on effect of xuesaitong injection in the treatment of acute cerebral injection. Chin Foreign Med Res. 2011;9(24):50-1.

33. Fu FC, Yang MY, Li JL, Cheng B, Li WJ. 122 cases of xuesaitong combined with sodium ozagrel on treating acute cerebral infarction. Guangming J Chin Mede. 2011;26(10):2087-8. 
34. He Q, Tan CB, Zhao Q. Clinical observation of xuesaitong injection combined with conventional treatment on patients with cerebral infarction. Drugs Clin. 2011;26(3):234-6.

35. Wang GY, Mao QL, Zhou ZP. Effect of xuesaitong injection on treating acute cerebral infarction and the influence on high sensitive C-reactive protein J Emerg Tradit Chin Med. 2011;20(4):639-40.

36. Yang $M$, Cheng YJ. 30 cases of xuesaitong injection combined with sodium ozagrel on treating acute cerebral infarction. China Mod Med. 2012;19(10):124.

37. Song Y. Observation on the effect of 80 cases of xuesaitong in the treatment of acute cerebral infarction. Chin J Urban Rural Ind Hyg. 2013;28(5):40-1.

38. Huang JS, Zhang ZM. Clinical studies on the relations of acute cerebral infarction and lipid. China Foreign Med Treat. 2011;30(15):45-6.

39. Stroke prevention guidelines writing group of Cerebrovascular Disease Study Group of Chinese Medical Association neuropathy credits. Primary prevention of stroke guide in Chinese 2010. Chin J Neurol. 2011;44(4):282-8.

40. Zhang X, Patel A, Horibe H, Wu Z, Barzi F, Rodgers A, et al. Cholesterol, coronary heartdisease, and stroke in the Asia Pacific region. Int J Epidemiol. 2003;32(4):563-72.

41. Kenarova B, Neychev H, Hadjiivanova C, Petkov VD. Immunomodulating activity of ginsenoside Rg1 from Panax ginseng. Jpn J Pharmacol. 1990;54(4):447-54

42. Chen WQ. Clinical value of detection FIB, CRP for elderly patients with acute cerebral infarction. For All Health. 2013;7(3):49-50.

43. Chen HY, Zhang LB, Jin RM. Meta-analysis of adverse reaction induced by xuesaitong injection. Pharmacol Clin Chin Materia Medica. 2012;28(2):163-6.

44. Duan CY. Systemic review on adverse reaction induced by xuesaitong injection. Jilin Med J. 2013;34(30):6264.

\section{Submit your next manuscript to BioMed Central and take full advantage of:}

- Convenient online submission

- Thorough peer review

- No space constraints or color figure charges

- Immediate publication on acceptance

- Inclusion in PubMed, CAS, Scopus and Google Scholar

- Research which is freely available for redistribution 